\title{
Enhancement of bistable perception associated with visual stimulus rivalry
}

\author{
Min-SuK Kang ANd Randolph BlaKe \\ Vanderbilt University, Nashville, Tennessee
}

\begin{abstract}
Rapid, repetitive exchange of dissimilar, rival stimuli between the two eyes can produce slow alternations in perceptual dominance. This phenomenon, called stimulus rivalry, is potentially important for studying resolution of visual conflict associated with neural processing beyond the level of interocular competition. As previously implemented, however, stimulus rivalry can be difficult for some observers to experience, and it tends to occur within a relatively narrow range of contrasts and spatial frequencies. Here we show that it is possible to increase the incidence of stimulus rivalry by brief, periodic presentation of a composite configuration created by superimposition of the two rival stimuli. Possible reasons for the effectiveness of the composite in promotion of stimulus rivalry are discussed.
\end{abstract}

To identify neural correlates of conscious visual awareness (Crick \& Koch, 1995), neuroscientists capitalize on bistable perception, a class of phenomena wherein visual experience fluctuates between alternative perceptual interpretations even though physical stimulation remains invariant (Andrews \& Purves, 1997; Blake \& Logothetis, 2002; Kim \& Blake, 2005; Leopold \& Logothetis, 1999). During bistable perception, there evidently exist modulations in neural activity coincident with the fluctuations in awareness, and identifying the sites of those neural modulations has become a challenge in singlecell recordings from awake, behaving animals (Leopold \& Logothetis, 1996; Logothetis \& Schall, 1989) and in human brain imaging (Haynes, Deichmann, \& Rees, 2005; Lumer, Friston, \& Rees, 1998; Polonsky, Blake, Braun, \& Heeger, 2000; Tong \& Engel, 2001; Wunderlich, Schneider, \& Kastner, 2005).

One very intriguing form of bistability can be provoked by presentation of two dissimilar visual figures to the two eyes, with the rival figures rapidly turned "on" and "off" and repeatedly swapped between the two eyes several times a second (Figure 1, left). This stimulus presentation regime can produce alternations in perception in which the dominance of a given figure lasts several seconds and, therefore, transcends multiple eye swaps (Logothetis, Leopold, \& Sheinberg, 1996). This form of bistability, unlike conventional binocular rivalry (Blake, 1989), cannot be explained by low-level interocular competition and, instead, implicates higher level neural competition between alternative stimulus representations (Wilson, 2003). However, this unique form of perceptual bistability, called stimulus rivalry, occurs within a rather narrow range of stimulus conditions (Bonneh, Sagi, \& Karni, 2001; Lee \& Blake, 1999), and, moreover, some observers experience difficulty in perceiving any bistability when viewing this eye-swapping display. Development of strategies for increasing the incidence of stimulus rivalry would promote its more widespread use as a tool for studying perceptual bistability, and that was the motive for the experiments described in this article.

The point of departure for our work was a concern about the possibly disruptive effect of the abrupt transients that occur as the gratings viewed by the two eyes change orientation every $333 \mathrm{msec}$. In their original article, Logothetis et al. (1996) were aware of this possibility, and they attempted "to minimize or eliminate the awareness of instances of stimulus reversals" (p. 623) by rapidly flickering $(18 \mathrm{~Hz})$ both gratings on and off throughout the period of stimulus presentation. Rapid flicker does indeed help perception of stimulus rivalry (Lee \& Blake, 1999), but it does not eliminate onset transients created when the gratings are swapped between the eyes. To minimize those transients, we modified the eye-swapping procedure so that the off periods of rival stimulation associated with the flicker/swap procedure (Figure 1, left) were replaced by repetitive presentation of the composite of the two rival grating patterns (Figure 1, right). We reckoned that the composite configuration might smooth the transition from one rival configuration to the other, while, at the same time, retaining the fast, repetitive flicker that facilitates perception of stimulus rivalry (Lee \& Blake, 1999).

We call this novel stimulus technique the composite/ swap sequence to distinguish it from the flicker/swap sequence characteristic of ordinary stimulus rivalry. In the experiments described below, we document that the composite/swap sequence enhances the incidence of stimulus rivalry for nearly all observers tested (Experiment 1), including under stimulus conditions where ordinary flicker/ swap sequences yield very few instances of slow alternations in perceptual dominance (Experiment 2).

M.-S. Kang, min-suk.kang@vanderbilt.edu 


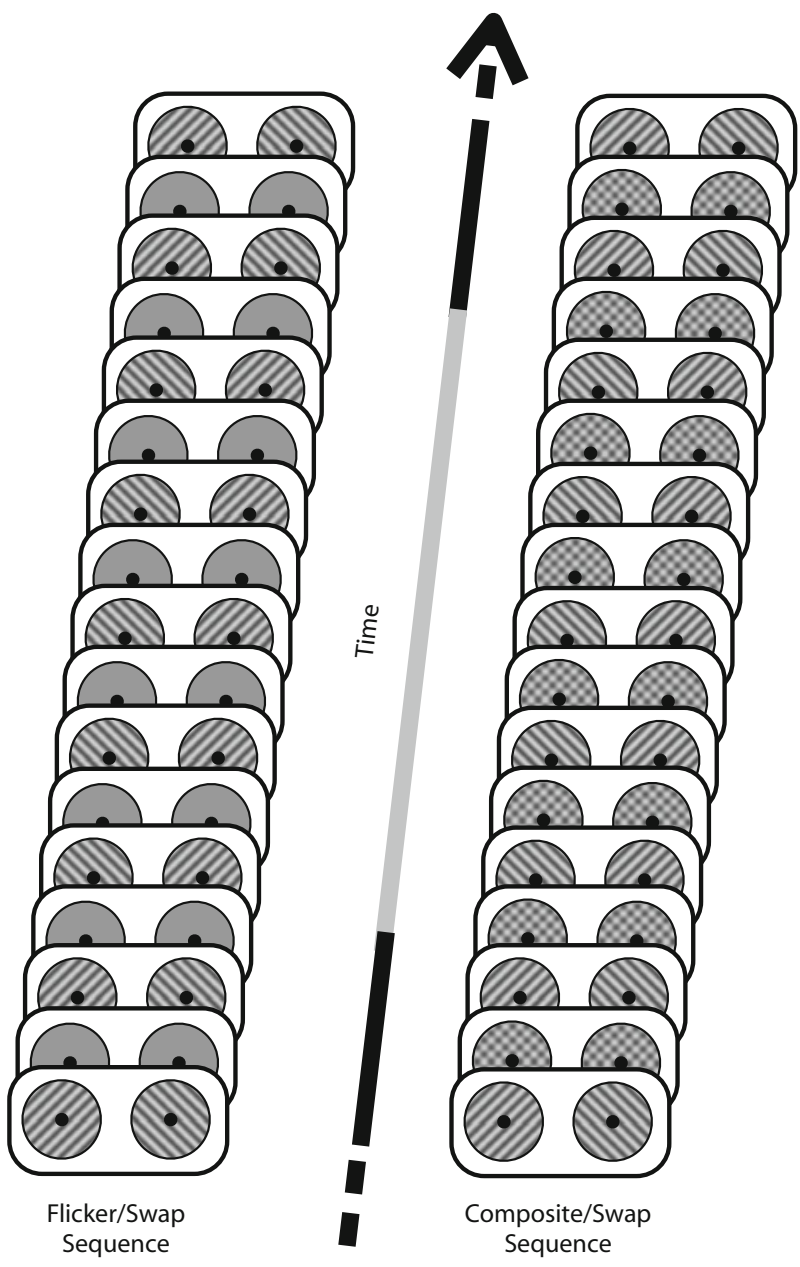

Figure 1. Schematic diagram of two forms of stimulus sequence tested in Experiments 1 and 2. When observers were viewing these sequences, perception slowly alternated distinctly as one of the two rival grating patterns was perceptually stronger for several seconds during the dominance phase. Observers were instructed to press and hold one of two keys only when the left-tilted grating or the right-tilted grating was distinctly dominant.

\section{EXPERIMENT 1}

We compared the incidence of stimulus rivalry using the composite/swap sequence and the flicker/swap sequence, the aim being to establish whether the composite/ swap sequence indeed enhances stimulus rivalry, including among observers who experience great difficulty in seeing stimulus rivalry when viewing the conventional flicker/swap sequence.

\footnotetext{
Method

Apparatus. Visual stimuli were created using MATLAB (MathWorks) in conjunction with the Psychophysics Toolbox (Brainard, 1997; Pelli, 1997). Stimuli were presented on the screen of a Mitsubishi Diamond Pro 2020u monitor (1,024 × 768 resolution; $120-\mathrm{Hz}$ frame rate) whose mean luminance was $24.22 \mathrm{~cd} / \mathrm{m}^{2}$ in a dimly lit room. A Macintosh G4 computer controlled all aspects of display presentation and testing. Rival stimuli were presented separately to each eye using a mirror stereoscope with eyepieces $90 \mathrm{~cm}$ from the monitor.
}

Stimuli and Procedure. The stimuli were round-windowed, sine-wave gratings, one oriented $45^{\circ}$ clockwise and the other $45^{\circ}$ counterclockwise. The spatial frequency of both rival stimuli was 3 cycles/deg and the contrast of both was $25 \%$. The diameter of the circular window was $2.4^{\circ}$ of visual angle, and the edges of both gratings were smoothed with a Gaussian filter whose full width at half maximum was $0.14^{\circ}$. A black circular frame (inner and outer diameters $2.7^{\circ}$ and $3.0^{\circ}$ ) surrounded each grating to promote stable binocular alignment, and a small, round fixation point was centered within both eyes' views to promote central fixation.

During the flicker/swap sequence, the two rival targets flickered rapidly on and off at $15 \mathrm{~Hz}$ and they were also swapped between the eyes every $333 \mathrm{msec}$. During the composite/swap sequence, off periods of the flicker/swap sequence were replaced by presentation of a plaid pattern created by multiplicative combination of the two rival targets. During prolonged viewing periods, observers pressed and held designated keys to indicate when one of the two rival gratings was distinctly dominant. Each trial lasted $30 \mathrm{sec}$ and each stimulus combination was tested four times.

To familiarize them with bistable perception, all observers were initially given a practice session during which they viewed conventional binocular rivalry in which the dissimilar monocular gratings were not swapped between the eyes. On some trials, those rival gratings were flickered on $/$ off at $15 \mathrm{~Hz}$, and on other trials, the off periods of flicker were replaced by presentation of the composite plaid to both eyes. In all other respects, these binocular rivalry displays, and the procedures used to measure rivalry, were identical to those used for the flicker/swap sequence and the composite/swap sequence described above.

Participants. Ten naive observers together with the first author of this article ( 9 male and 2 female) participated in this experiment. All had normal or corrected-to-normal vision, and all gave informed consent after thorough explanation of the procedures.

\section{Results}

The normalized frequency distributions produced by the flicker/swap sequence and the composite/swap sequence were unimodal and skewed toward longer durations (Figure 2A). Comparison of these two normalized distributions using the Kolmogorov-Smirnov test reveals that they are not significantly different $(p=.57)$. These histograms conform to the general shape of the frequency distributions associated with a variety of bistable phenomena (see, e.g., Brascamp, van Ee, Pestman, \& van den Berg, 2005) including stimulus rivalry (Logothetis et al., 1996). We conclude, therefore, that including the composite stimulus in the eye-swap procedure does not alter the stochastic properties of the perceptual bistability experienced when one is viewing the sequence. But does the composite influence the incidence of stimulus rivalry?

Figure $2 \mathrm{~B}$ compares the two sequence procedures by plotting for each observer the incidence of stimulus rivalry from the flicker/swap sequence against the incidence of stimulus rivalry for the composite/swap sequence. (Incidence is defined as the total time for which either grating was distinctly dominant, divided by the total viewing period.) Notice that the data points tend to fall above the diagonal line, indicating that observers experience stimulus rivalry more frequently when viewing the composite/swap sequence $[t(10)=2.47, p<.05]$. Note, too, the pair of data points lying very near the $y$-axis - these data came from 2 observers who almost never experienced slow alternations in dominance when viewing the conventional flicker/swap sequence but did perceive stimulus rivalry when viewing 

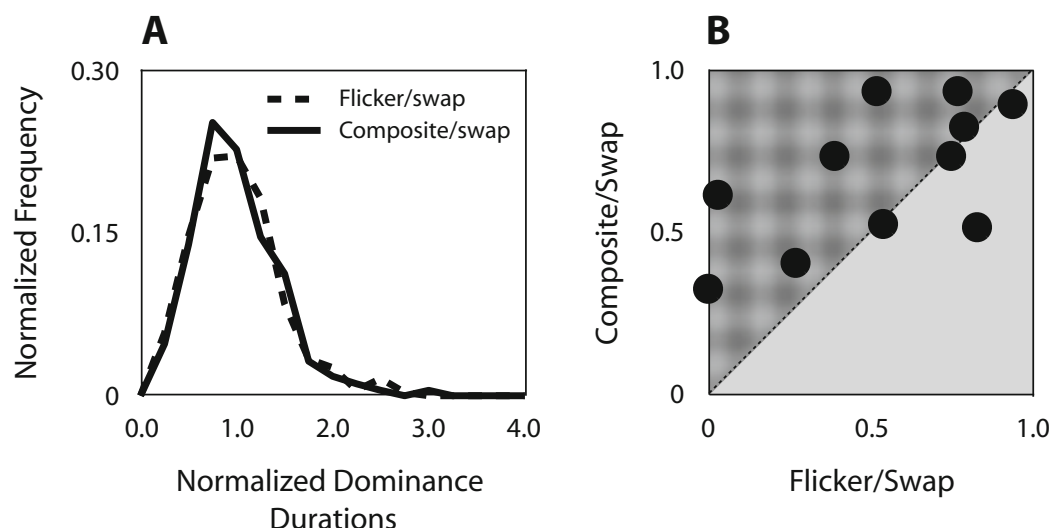

Figure 2. Comparison of the incidence of stimulus rivalry for two kinds of presentation sequences. (A) Normalized frequency histogram of stimulus rivalry from the composite/swap sequence (solid line) and flicker/swap sequence (dashed line). For each observer, dominance durations were normalized by that individual's mean dominance duration. (B) Scatterplot of stimulus rivalry incidence for viewing flicker/ swap sequence and for viewing composite/swap sequence. Each point indicates the incidence of stimulus rivalry of a single observer tested on both conditions.

the composite/swap sequence. It is also noteworthy that 9 of 11 observers tested in this experiment had never been exposed to stimulus rivalry before these data were collected (this includes the single observer, obvious in Figure 2B, for whom the flicker/swap sequence was more effective).

The enhanced incidence of stimulus rivalry associated with the composite/swap sequence led us next to ask whether this novel technique could expand the range of stimulus conditions under which bistable perception is experienced. To pursue that question, we performed a second experiment.

\section{EXPERIMENT 2}

This experiment compared the incidence of stimulus rivalry produced by the composite/swap and the flicker/swap sequences comprising rival targets whose contrast and spatial frequency were varied independently. We focused on these stimulus dimensions because previous work (Lee \& Blake, 1999) showed that stimulus rivalry rarely occurs when target contrast is high or when spatial frequency is low.

\section{Method}

Stimuli and Procedure. For the contrast condition, four contrast values $(20 \%, 30 \%, 40 \%$, and $50 \%)$ were tested with spatial frequency held constant at 3 cycles/deg. For the spatial frequency condition, four spatial frequencies $(1.2,2.4,3.6$, and 4.8 cycles $/ \mathrm{deg})$ were tested with contrast held constant at $25 \%$. All other aspects of the stimuli and procedure were identical to those in Experiment 1. Variations in contrast and in spatial frequency were tested in separate sessions. A single trial lasted $30 \mathrm{sec}$ and was repeated four times for each condition. Each session consisted of 32 randomly intermixed trials (4 levels of a given stimulus dimension $\times 2$ types of swap sequence $\times 4$ repetitions).

Participants. Six observers (5 male and 1 female), including the first author, participated in this experiment. All 6 had participated in Experiment 1.

\section{Results}

As shown in Figures 3A and 3B, when the flicker/swap sequence was viewed, the incidence of stimulus rivalry decreased as the contrast of rival stimuli increased or as the spatial frequency of the stimuli decreased. This pattern of results replicates previous work (Lee \& Blake, 1999). Although still dependent on spatial frequency and contrast, the total amount of time that stimulus rivalry was experienced increased when the composite/swap sequence was viewed. A repeated measures ANOVA showed that the mean dominance durations for viewing the composite/ swap sequence were approximately $50 \%$ longer than the mean dominance durations for viewing the flicker/swap sequence [contrast condition, $F(1,5)=27.60, p<.01$; spatial frequency, $F(1,5)=7.30, p<.05$ ]. (See Tables 1 and 2.) This implies that perceptual dominance is more resistant to disruptions instigated by the eye-swapping procedure when the composite/swap sequence is viewed.

Could this increased incidence of stimulus rivalry associated with the composite/swap sequence be attributed to neural adaptation produced by the repeated exposures to the composite configuration? The composite configuration presumably activates neurons responsive to the component orientations of that composite, whereas with the flicker/swap sequence, those neurons would not be activated during the off periods. Over time, then, orientationselective neurons would be activated for a longer total amount of time during the composite sequence, and, therefore, those neurons would be more likely to adapt. It is known that adaptation generally weakens the effective stimulus strength of a pattern (Blakemore \& Campbell, 1969; Hammett, Snowden, \& Smith, 1994), and we know that the incidence of stimulus rivalry is greater when stimulus strength is weak (Lee \& Blake, 1999).

To evaluate the potential contribution of adaptation, we calculated the incidence of stimulus rivalry during the first 10-sec epoch and during the last 10-sec epoch of the 30-sec tracking period of both the composite/swap and the flicker/swap sequence. The logic was as follows: If adaptation causes the higher incidence of stimulus ri- 
A

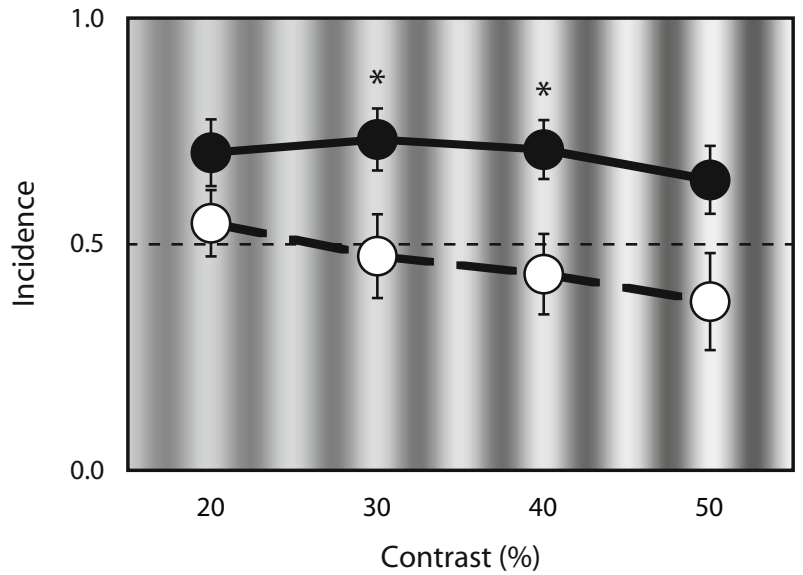

B

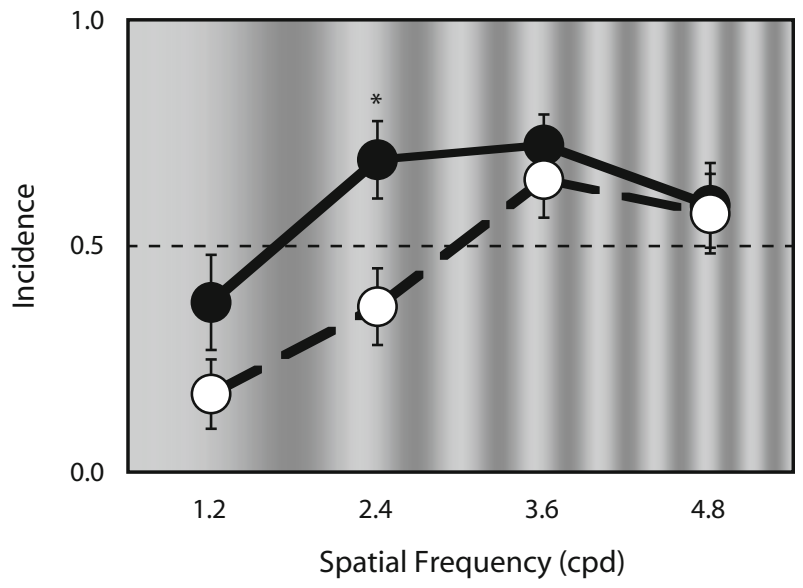

Figure 3. Comparison of the incidence of stimulus rivalry for two kinds of presentation sequences tested at different contrast values (A) and different spatial frequency values (B). Asterisks indicate differences between sequence type where $p<.05$ when difference $t$ tests were performed for each contrast and spatial frequency level. Filled circles connected by a solid line indicate the stimulus rivalry from composite/swap sequence; open circles connected with a dotted line indicate the stimulus rivalry from flicker/swap sequence. Error bars equal one standard error. (A) The incidence of stimulus rivalry for viewing the composite/ swap sequence was higher than the incidence for viewing the flicker/swap sequence at overall contrast levels $[F(1,5)=7.11$, $p<.05$; repeated measures ANOVA]. (B) The incidence of stimulus rivalry at high spatial frequencies ( 3.6 and 4.8 cycles/deg) was equivalent for the two sequences, but the incidence of stimulus rivalry for viewing composite/swap sequence was higher at low spatial frequency levels in comparison with the flicker/swap condition (1.2 and 2.4 cycles/deg).

valry when viewing the composite/swap sequence, we should observe a differential effect of this swap sequence during the last tracking epoch, when adaptation would have grown in magnitude. This would be evidenced by a statistically significant two-way interaction between stimulus condition - the composite/swap sequence and the flicker/swap sequence - and epoch - the first $10 \mathrm{sec}$ and the last $10 \mathrm{sec}$. Repeated measures ANOVAs revealed no such evidence, however, for either the contrast condition $[F(1,5)=2.60, p=.17]$ or the spatial frequency condition $[F(1,5)=5.90, p=.06]$. The same lack of significance for the condition $\times$ epoch interaction was found when a repeated measures ANOVA was performed on the average dominance durations during the first versus the last $10 \mathrm{sec}$ [contrast condition, $F(1,5)=3.74$, $p=.11$; spatial frequency condition, $F(1,5)=2.31, p=$ .18]. We conclude, therefore, that the enhanced incidence of stimulus rivalry when composite/swap sequences are viewed cannot be attributed to differential adaptation during the composite configuration.

\section{DISCUSSION}

In two experiments, we found that stimulus rivalry occurs more readily when accompanied by exposure of both eyes to a nonrival composite of both rival targets. When the off period of conventional flicker/swap is replaced with this dioptic, composite configuration, the incidence of stimulus rivalry increases for most observers and is experienced over a wider range of stimulus conditions. By what means does the composite enhance perception of stimulus rivalry?

Our initial hypothesis was that presence of the composite might enhance stimulus rivalry by minimizing the onset transients caused by the abrupt changes in orientation produced by the eye-swap procedure. The two panels in Figure 4 schematically illustrate what we have in mind. In the flicker/swap sequence (Figure 4A), rival stimulation is immediately preceded by a period during which both eyes see uncontoured regions of average luminance. This means, therefore, that the onset of rival stimulation is abrupt. Under at least some circumstances, visual transients can disrupt rivalry (Fox \& Check, 1968; P. Walker \& Powell, 1979). Recall, too, that Logothetis et al. (1996) attempted to minimize the consequences of abrupt stimulus reversals by embedding those reversals in a constant stream of rapid flicker. With the composite/swap sequence (Figure 4B), both orientation components constituting the period of rival stimulation are present in the two eyes immediately preceding this rival period. The only transient immediately preceding rival stimulation is created by the offset of one of the two component orientations, and that transient is minimized because the other component remains present in that eye.

The composite's effectiveness may also arise from its engagement of monocular rivalry, the reciprocal fluctuations in the clarity of two different, superimposed images such as orthogonally oriented gratings (Breese, 1899). ${ }^{1}$ Two lines of evidence suggest that monocular rivalry from the composite could enhance stimulus rivalry. First, Pearson and Clifford (2005) have shown that monocular rivalry can indeed interact with stimulus rivalry and, for that matter, with binocular rivalry, to promote coherent perceptual dominance over space and time. Second, Atkinson, Campbell, Fiorentini, and Maffei (1973) have shown that the incidence of monocular rivalry increases at lower contrasts and at lower spatial frequencies, which is also the consequence of the composite in our experiments (Figure 3). At present we do not know the neural bases of monocular rivalry, although it is tempting to think 
Table 1

Mean Dominance Durations (in Seconds) of Stimulus Rivalry for Each Contrast Level

\begin{tabular}{lllll}
\hline & \multicolumn{4}{c}{ Contrast Level } \\
\cline { 2 - 5 } & $20 \%$ & $30 \%$ & $40 \%$ & $50 \%$ \\
\hline Flicker/swap & 1.51 & 1.27 & 1.33 & 1.11 \\
Composite/swap & 2.32 & 1.96 & 1.82 & 1.87 \\
\hline
\end{tabular}

Table 2

Mean Dominance Durations (in Seconds) of Stimulus Rivalry for Each Spatial Frequency Level

\begin{tabular}{lcccc}
\hline & \multicolumn{4}{c}{ Spatial Frequency (cycles/deg) } \\
\cline { 2 - 5 } & 1.2 & 2.4 & 3.6 & 4.8 \\
\hline Flicker/swap & 1.15 & 1.06 & 1.59 & 1.60 \\
Composite/swap & 1.46 & 2.04 & 2.06 & 2.14 \\
\hline
\end{tabular}

about the phenomenon in terms of cross-orientation inhibition (Morrone, Burr, \& Maffei, 1982), the decrease in a visual neuron's response to its preferred stimulus when a second, nonpreferred stimulus is superimposed on the preferred one (Baker, Meese, \& Summers, 2007; Bonds, 1989; Carandini, Heeger, \& Movshon, 1997; G. A. Walker, Ohzawa, \& Freeman, 1998).

What are the implications of our results for models of rivalry? A number of neural models have been proposed to account for conventional binocular rivalry, wherein the dissimilar stimuli are not swapped between the eyes (Blake, 1989; Laing \& Chow, 2002; Lehky, 1988). Only the model developed by Wilson (2003), however, is designed to simulate both conventional binocular rivalry and stimulus rivalry, wherein stimuli are swapped between the eyes. Even the Wilson model, however, was not constructed to accommodate the novel stimulus conditions used in our experiments. Specifically, his model does not include the circuitry needed to represent the binocularly matched components of the composite figure; in the Wilson model, those components can only compete with one another, just as do the rival targets. Although beyond the scope of the present article, it would be useful to explore ways to merge Wilson's competitive interaction network with extant models of

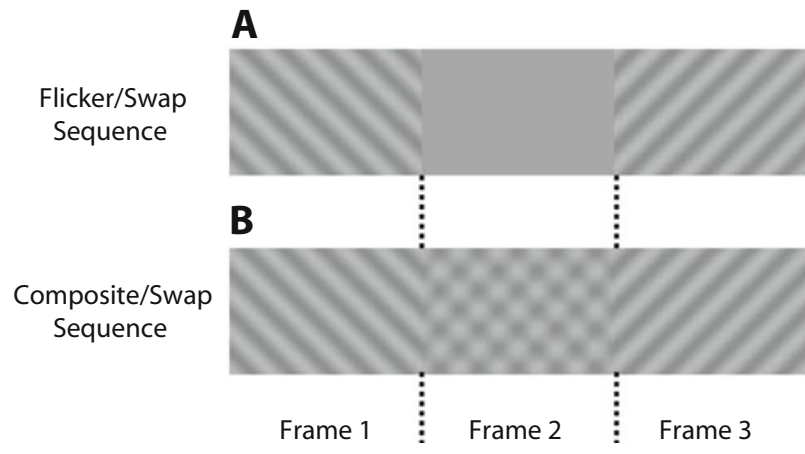

Figure 4. Schematics illustrating three successive display frames of the flicker/swap sequence (A) and the composite/swap sequence $(B)$. binocular combination, such as the gain-control model of Ding and Sperling (2006).

\section{AUTHOR NOTE}

This work was supported by National Institutes of Health Grant EY13358. We thank Hugh Wilson for helpful discussion and Chai-Youn Kim, Joel Pearson, and Eunice Yang for comments on a previous version of the manuscript. Correspondence concerning this article should be addressed to M.-S. Kang, Department of Psychology, Vanderbilt University, Wilson Hall, 11121 st Avenue South, Nashville, TN 37203 (e-mail: min-suk.kang@vanderbilt.edu).

\section{REFERENCES}

Andrews, T., \& Purves, D. (1997). Similarities in normal and binocularly rivalrous viewing. Proceedings of the National Academy of Sciences, 94, 9905-9908.

Atkinson, J., Campbell, F. W., Fiorentini, A., \& Maffei, L. (1973). The dependence of monocular rivalry on spatial frequency. Perception, 2, 127-133.

Baker, D. H., Meese, T. S., \& Summers, R. J. (2007). Psychophysical evidence for two routes to suppression before binocular summation of signals in human vision. Neuroscience, 146, 435-448.

Blake, R. (1989). A neural theory of binocular rivalry. Psychological Review, 96, 145-167.

Blake, R., \& Logothetis, N. K. (2002). Visual competition. Nature Reviews Neuroscience, 3, 13-21.

Blakemore, C., \& Campbell, F. W. (1969). On the existence of neurones in the human visual system selectively sensitive to the orientation and size of retinal images. Journal of Physiology, 203, 237-260.

Bonds, A. B. (1989). Role of inhibition in the specification of orientation selectivity of cells in the cat striate cortex. Visual Neuroscience, 2, 41-55.

Bonneh, Y., SAGi, D., \& Karni, A. (2001). A transition between eye and object rivalry determined by stimulus coherence. Vision Research, 41, 981-989.

Brainard, D. H. (1997). The Psychophysics Toolbox. Spatial Vision, 10, 433-436.

Brascamp, J. W., van Ee, R., Pestman, W. R., \& van den Berg, A. V. (2005). Distributions of alternation rates in various forms of bistable perception. Journal of Vision, 5, 287-298.

Breese, B. B. (1899). On inhibition. Psychological Monographs, 3, $1-65$.

Carandini, M., Heeger, D. J., \& Movshon, J. A. (1997). Linearity and normalization in simple cells of macaque primary visual cortex. Journal of Neuroscience, 17, 8621-8644.

CRICK, F., \& КосH, C. (1995). Are we aware of neural activity in primary visual cortex? Nature, 375, 121-123.

Ding, J., \& SPERLING, G. (2006). A gain-control theory of binocular combination. Proceedings of the National Academy of Sciences, 103, $1141-1146$

Fox, R., \& CHecK, R. (1968). Detection of motion during binocular rivalry suppression. Journal of Experimental Psychology, 78, 388-395.

Hammett, S. T., Snowden, R. J., \& Smith, A. T. (1994). Perceived contrast as a function of adaptation duration. Vision Research, 34, 31-40.

Haynes, J.-D., Deichmann, R., \& Rees, G. (2005). Eye-specific effects of binocular rivalry in the human lateral geniculate nucleus. Nature, 438, 496-499.

Kim, C. Y., \& Blake, R. (2005). Psychophysical magic: Rendering the visible "invisible." Trends in Cognitive Sciences, 9, 381-388.

LAING, C. R., \& CHOW, C. C. (2002). A spiking neuron model for binocular rivalry. Journal of Computational Neuroscience, 12, 39-53.

LeE, S.-H., \& BlaKe, R. (1999). Rival ideas about binocular rivalry. Vision Research, 39, 1447-1454.

LEHKY, S. R. (1988). An astable multivibrator model of binocular rivalry. Perception, 17, 215-228.

LeOPOLD, D. A., \& Logothetis, N. K. (1996). Activity changes in early visual cortex reflect monkeys' percepts during binocular rivalry. $\mathrm{Na}$ ture, 379, 549-553. 
Leopold, D. A., \& Logothetis, N. K. (1999). Multistable phenomena: Changing views in perception. Trends in Cognitive Sciences, $\mathbf{3}$, 254-264.

Logothetis, N. K., Leopold, D. A., \& Sheinberg, D. L. (1996). What is rivalling during binocular rivalry? Nature, 380, 621-624.

Logothetis, N. K., \& Schall, J. D. (1989). Neuronal correlates of subjective visual perception. Science, 245, 761-763.

Lumer, E., Friston, K., \& ReEs, G. (1998). Neural correlates of perceptual rivalry in the human brain. Science, 280, 1930-1934.

Morrone, M. C., Burr, D. C., \& Maffei, L. (1982). Functional implications of cross-orientation inhibition of cortical visual cells: I. Neurophysiological evidence. Proceedings of the Royal Society of London B, 216, 335-354.

Pearson, J., \& Clifford, C. W. (2005). When your brain decides what you see: Grouping across monocular, binocular, and stimulus rivalry. Psychological Science, 16, 516-519.

Pelli, D. G. (1997). The VideoToolbox software for visual psychophysics: Transforming numbers into movies. Spatial Vision, 10, 437-442.

Polonsky, A., Blake, B., Braun, J., \& Heeger, D. J. (2000). Neuronal activity in human primary visual cortex correlates with perception during binocular rivalry. Nature Neuroscience, 3, 1153-1159.

Tong, F., \& ENGEL, S. A. (2001). Interocular rivalry revealed in the human cortical blind-spot representation. Nature, 411, 195-199.
Walker, G. A., Ohzawa, I., \& Freeman, R. D. (1998). Binocular cross-orientation suppression in the cat's striate cortex. Journal of Neurophysiology, 79, 227-239.

Walker, P., \& Powell, D. J. (1979). The sensitivity of binocular rivalry to changes in the nondominant stimulus. Vision Research, 19, 247-249.

WiLson, H. R. (2003). Computational evidence for a rivalry hierarchy in vision. Proceedings of the National Academy of Sciences, 100, 14499-14503.

Wunderlich, K., Schneider, K. A., \& Kastner, S. (2005). Neural correlates of binocular rivalry in the human lateral geniculate nucleus. Nature Neuroscience, 8, 1595-1602.

\section{NOTE}

1. This form of perceptual bistability is called monocular because it does not require dichoptic presentation of the two components. Note, however, that "monocular" does not mean that the two gratings must be viewed with one eye only; binocular viewing can yield compelling monocular rivalry.

(Manuscript received July 24, 2007; revision accepted for publication December 11, 2007.) 